Article

\title{
Event-Based Quantum Mechanics: A Context for the Emergence of Classical Information
}

\author{
Ignazio Licata ${ }^{1,2,3, *}$ and Leonardo Chiatti ${ }^{4, *}$ \\ 1 School of Advanced International Studies on Applied Theoretical and Non Linear Methodologies in Physics, \\ 70121 Bari, Italy \\ 2 Institute for Applicable Mathematics and Information Sciences, B.M. Birla Science Centre, Adarsh Nagar, \\ 500063 Hyderabad, India \\ 3 Research Institute for Astronomy and Astrophysics of Maragha, Valeasr, 5517736698 Maragheh, Iran \\ 4 ASL VT Medical Physics Laboratory, Via Enrico Fermi 15, 01100 Viterbo, Italy \\ * Correspondence: ignazio.licata3@gmail.com (I.L.); leonardo.chiatti@asl.vt.it (L.C.)
}

Received: 7 January 2019; Accepted: 31 January 2019; Published: 3 February 2019

\begin{abstract}
This paper explores an event-based version of quantum mechanics which differs from the commonly accepted one, even though the usual elements of quantum formalism, e.g., the Hilbert space, are maintained. This version introduces as primary element the occurrence of micro-events induced by usual physical (mechanical, electromagnetic and so on) interactions. These micro-events correspond to state reductions and are identified with quantum jumps, already introduced by Bohr in his atomic model and experimentally well established today. Macroscopic bodies are defined as clusters of jumps; the emergence of classicality thus becomes understandable and time honoured paradoxes can be solved. In particular, we discuss the cat paradox in this context. Quantum jumps are described as temporal localizations of physical quantities; if the information associated with these localizations has to be finite, two time scales spontaneously appear: an upper cosmological scale and a lower scale of elementary "particles". This allows the interpretation of the Bekenstein limit like a particular informational constraint on the manifestation of a micro-event in the cosmos it belongs. The topic appears relevant in relation to recent discussions on possible spatiotemporal constraints on quantum computing.
\end{abstract}

Keywords: Bekenstein information; emergent time; localization; quantum jump; quantum measurements

\section{Introduction}

Quantum mechanics (QM) is the current theory of reference in the study of the micro-physical world. The application of its principles led to the full elucidation of the behavior of matter at the particle scale, of the atomic nuclei, of atoms, of molecules, of condensed matter. Such principles are at the base of the prodigious development of today's quantum technologies. In addition, it was the starting point for the formulation of quantum field theory. Nevertheless, the nature of the physical world at the scale of quantum processes remains the subject of debate [1]. In particular, the mechanisms that convert the quantum information associated with these processes into classical information, thus allowing the emergence of the macroscopic classical world, remain elusive. In this article we wish to investigate these mechanisms in a new light. In order to illustrate the approach, let us consider an unstable microscopic quantum system, let us say an atomic nucleus of Radium 226 in isolation. This system decays in Radon 222 with a very long half-life (1600 years); this means that the nuclear quantum amplitude undergoes a slow unitary time evolution tending asymptotically to the amplitude of the nuclear state designated as Radon 222. This description would potentially be applicable both to the single nucleus of Radium 226 and to a set of nuclei of Radium 226 prepared at the same initial 
moment. Now, the peculiarity of QM is that the amplitude of a single nucleus of Radium 226 can undergo a discontinuous jump to the amplitude representative of the Radon 222. This discontinuous evolution is what is called a quantum jump (QJ).

It is noteworthy, first of all, that a $Q J$ is an event in the history of a single nucleus; identical nuclei simultaneously prepared in the same way will decay at different times. Therefore, the time evolution of the set of all these nuclei will lead to a mixture consisting of a decreasing fraction of non-decayed nuclei (whose amplitudes will be expressed by the same superposition of Radium 226 and Radon 222) and a growing fraction of Radon 222. Moreover the decay, that is the $Q J$, is an objective physical fact. Indeed a detector placed near the nucleus that undergoes the decay will detect an alpha particle, product of the decay (and possibly the gamma photon of rearrangement). By applying the principles of conservation of physical quantities such as charge, energy and impulse, we obtain that the quantities transported by the decay products are those related to the transformation of Radium 226 in Radon 222. Since the emission of these products is instantaneous with respect to the unitary evolution of the nuclear amplitude, it follows that the $Q J$ is instantaneous on the scale of this second process (and it can be assumed that it is instantaneous in the strict sense).

Therefore, there is an objective physical process, the QJ, which converts quantum information, associated with a superposition, in classical information associated with a mixture. This process operates on a microscopic scale, therefore in the absence of any noise that can produce decoherence and in total isolation. Furthermore, it does not depend on the presence or absence of measurement devices (which can reveal at most the decay products when the process has ended). The QJ is clearly an outcome of the known and usual physical interactions: specifically, the strong nuclear interaction governing the alpha decay. These interactions produce two effects: a unitary evolution of the amplitudes that can be described in principle by the quantum equations of motion and their discontinuous and non-unitary variation constituted by the QJ. In other words, each interaction induces both Hamiltonian and non-Hamiltonian effects on the evolution of quantum amplitudes. The quantum jumps were introduced by Bohr in his famous trilogy [2-4], in relation to the explanation of atomic spectral lines. Direct observation of atomic QJs and their discrimination from the underlying unitary process required the development of refined experimental methods that became available in the mid 1980s. With the method of ionic traps was possible to use "shelved" atoms for this observation [5]. Subsequently, quantum jumps were directly observed in a number of atomic, molecular, electromagnetic and nuclear microsystems [6-19].

However, while the QJs represent a well established experimental fact, the current formulations of the QM are not based on the explicit recognition of their existence. It is good to repeat that $Q J$ is a real physical phenomenon (for example, the effective transmutation of an atomic nucleus with the emission of observable decay products) that occurs as the effect of a specific interaction (in the example, a strong interaction). It should not be confused with its effect, i.e., the reduction of quantum amplitude and the consequent production of classical information. Furthermore, while this information may become available as a measure of the knowledge acquired by a human observer, the presence of such an observer is not a necessary condition for the QJ to take place. We take the QJs as elementary "events" of an objective physical world, independent of the presence of observers. Furthermore, we adopt an ontological perspective where the macroscopic systems are stable aspects of recurrent schemes of QJs. The QM, reformulated according to these principles, thus becomes a description of the physics of this objective world. Now we want to discuss some of the advantages of this perspective.

Since the QJs induce a reduction of quantum amplitude, one may question if they may have some relationship with the other phenomenon to which the QM attributes the same property, that is, the measurement. In this paper we will assume that the reduction induced by the quantum measurement process is nothing but the effect of a quantum jump on the evolution of the complex consisting of the measurement apparatus and the micro-entity. For consistency with the previous reasoning, the jump will be the non-Hamiltonian effect of the (even negative) interaction between the apparatus and the micro-entity. In this way the measurement becomes an ordinary physical phenomenon and we can 
consider it as a special case of QJ. The concepts of "measurement" and "observable" therefore lose their centrality in the construction of the theory. The peculiarities of quantum measurement will be examined later.

The succession of the QJs experienced over time by the same elementary "quantum system" is naturally a discrete set: two successive QJs are separated by a finite time interval. The lack of existence of events related to the system in this interval means that in this interval the "system", intended as a set of QJs, does not exist in time. The elementary "quantum system" is therefore not an object, in the classical sense: it is a causal connection between single QJs. Its identity will be characterized by the existence of a specific Hilbert space where the transition amplitude between a QJ and the next is evaluated (if the number of particles is not conserved, we will have instead a Fock space, but in this paper we will not discuss this circumstance). The quantum amplitude (bra or ket) associated with a system is therefore not the "state" of some object; in particular, a superposition of amplitudes should not be intended as a superposition of "states". The situation is very different if the "quantum system" is not elementary, that is, it consists of a multiplicity of elementary constituents able to interact between themselves with production of many QJs at the same time. The characterization of the system will then also require the specification of these QJs. The case of classical macro-systems is that of the ideal limit in which the system is defined entirely by average properties, relatively stable over time, of an enormously high number of QJs. Such a system is an object, it is endowed with continuous existence in time and its state is described by classical variables, to which classical measures of information are attributable.

By attributing the role of primary constituents of the physical world on the quantum scale to the QJs is therefore possible to construct a version of the QM in which the transition from the (fundamental) quantum level to the classical one is fully understandable. To construct such a version we have to remember that a $Q J$ projects the quantum amplitude of the system on a given subspace of the Hilbert space; it can therefore be represented by a self-conjugated operator. The $Q J$ is therefore the localization, in the temporal domain, of that projection and of the physical quantities associated with it. Temporal localizations of physical quantities thus assume the role of primary elements of physical reality, with the result that this one is made up of events, and no longer objects.

This formulation assigns the same theoretical and experimental significance to two inseparable and complementary aspects of the quantum world: that localized in time (QJs) and that not localized in time (amplitudes). The relationship between these two aspects can be described in terms of the relationship between quantum information coded in the Hilbert space and classical information produced in the QJs. In particular, the Bekenstein limit becomes a limit on classical information which can be expressed in a finite volume of space by the QJs. If this limit has to be finite, the temporal de-localization must be limited both above, on the cosmological scale, and below. This last constraint determines the appearance of a scale of elementary particles, an element which is not explicit in the usual formulation of the QM. The evolution of the superposition of amplitudes in a quantum computer, however, does not happen in spacetime. Therefore, this evolution does not seem to be limited by spatio-temporal constraints, which instead can afflict the input-output operations. The recent debate on the possibilities of quantum computing raised by Davies and Aaronson [20,21] should be considered in this perspective.

The structure of this paper is as follows. In Section 2, the quantum basic notions are re-arranged according to the principles outlined in this introduction; in particular the notion of event is specified and the Born rule is introduced. Section 3 briefly introduces the notions of classical system and measurement. Section 4 is dedicated to the discussion of the cat paradox. In Section 5 we discuss the relationship between localization and information. In Section 6, some considerations about the possible spatiotemporal limits of quantum computing are discussed according to the perspective illustrated in this paper. A comparison with other similar approaches in the literature is reported in Section 7. Open problems and the possibility of future research are briefly sketched in Conclusions. 


\section{Rewriting Quantum Postulates}

In this section we present a reformulation of the basic concepts of the QM according to the recognition of the fundamental nature of the QJs as events on the quantum scale. In order to highlight the impact of this recognition, we will refer to the semi-formalized presentation level typical of textbooks. In our intervention on the standard formalism we will try to be as conservative as possible, compatibly with the variations we intend to represent.

We assume two primitive theoretical concepts:

(1) A real variable $t \in T \subset \mathbb{R}$, the 'time'.

(2) A 'rigged' Hilbert space $\mathbf{H}$ with a scalar product $\langle\varphi \mid \psi\rangle \in \mathbb{C} ;|\psi\rangle,|\varphi\rangle \in \mathbf{H}$.

Postulate 1. $\exists E \subset T$ such that:

(a) $E$ is finite or countable;

(b) $\forall t_{k} \in E, k \in \mathbb{N}, \exists ! f: t_{k} \rightarrow\left|\psi_{t_{k}}\right\rangle\left\langle\psi_{t_{k}}|;| \psi_{t_{k}}\right\rangle \in \mathbf{H}$

(c) For each value of $k, a$ conditional probability $\operatorname{Prob}\left[\left(\left|\psi_{t_{k}}\right\rangle\left\langle\psi_{t_{k}}\right|\right) \|\left(\left|\psi_{t_{k+1}}\right\rangle\left\langle\psi_{t_{k+1}}\right|\right)\right]=$ $\operatorname{Prob}\left[\left(\left|\psi_{t_{k+1}}\right\rangle\left\langle\psi_{t_{k+1}}\right|\right) \|\left(\left|\psi_{t_{k}}\right\rangle\left\langle\psi_{t_{k}}\right|\right)\right]$ exists.

We call the application $f$ the 'manifestation' of the 'event' $\left|\psi_{t_{k}}\right\rangle\left\langle\psi_{t_{k}}\right|$. As one can see, this event is a self-adjoint operator on $\mathbf{H} ; t_{k}$ is the 'instant' when the event 'occurs'. It is assumed that $t_{k+1} \geq t_{k}$, $\forall k \in \mathbb{N}$ and there are not events manifested in the interval $\left(t_{k}, t_{k+1}\right)$. The postulate 1 thus defines a causal structure of successive events based on a conditional probability. Let us consider now a linear operator $\widehat{o}$ on $\mathbf{H}$, which is diagonal in the orthonormed and complete basis $\left|\phi_{i}\right\rangle ; i=1,2,3 \ldots$ :

$$
\widehat{o}=\Sigma_{i} o_{i}\left|\phi_{i}\right\rangle\left\langle\phi_{i}\right|
$$

The projector $\left|\phi_{i}\right\rangle\left\langle\phi_{i}\right|$ is a possible image of the application $f$ at the time $t$ if $\exists|\psi\rangle \in \mathbf{H}$ such that $\operatorname{Prob}\left[\left(\left|\psi_{t^{\prime}}\right\rangle\left\langle\psi_{t^{\prime}}\right|\right) \|\left(\left|\phi_{i, t}\right\rangle\left\langle\phi_{i, t}\right|\right)\right] \not \equiv 0$, where $t^{\prime} \in T$. If $o_{i} \in \mathbb{R}$ and each $\left|\phi_{i}\right\rangle\left\langle\phi_{i}\right|$ is a possible image of the application $f$ then $\widehat{o}$ is called 'physical quantity' on the space $\mathbf{H}$.

Postulate 2. The conditional probability of two successive events $\left|\psi_{t_{k}}\right\rangle\left\langle\psi_{t_{k}}\right|$ and $\left|\psi_{t_{k+1}}\right\rangle\left\langle\psi_{t_{k+1}}\right|$ is expressed as:

$$
P=\left\langle\psi_{t_{k+1}}|\widehat{S}| \psi_{t_{k}}\right\rangle\left\langle\psi_{t_{k}}|\widehat{S}| \psi_{t_{k+1}}\right\rangle
$$

with

$$
\widehat{S}=\exp \left(-\frac{i}{\hbar} \int_{t_{k}}^{t} \widehat{H} d \sigma\right) ; \sigma \in\left(t_{k}, t_{k+1}\right)
$$

where $\widehat{H}=\widehat{H}^{+}$is a physical quantity on $\mathbf{H}$ called 'Hamiltonian'.

Postulate 2 defines a rule for the conditional probability that connects two subsequent events (Born rule). The mean value of the physical quantity (1) manifested in the event $\left|\psi_{t_{k}}\right\rangle\left\langle\psi_{t_{k}}\right|$ is given, as it is easy to verify, by:

$$
\langle\widehat{o}\rangle=\operatorname{Tr}\left[\left|\psi_{t_{k}}\right\rangle\left\langle\psi_{t_{k}}\right| \widehat{o}\right]
$$

The postulate 2 does not privilege either of the two directions of time. However, it is also possible to introduce a time-oriented dynamics by defining the pair of amplitudes:

$$
\begin{array}{r}
\left|\psi_{t}^{\prime}\right\rangle=\widehat{S}\left|\psi_{t_{k}}\right\rangle ; \text { quantum 'forward' amplitude } \\
\left\langle\psi_{t}^{\prime \prime}\right|=\left\langle\psi_{t_{k+1}}\right| \widehat{S}^{+} ; \text {quantum 'backward'amplitude }
\end{array}
$$

to which the two time evolution equations, equivalent to Equation (2):

$$
\begin{aligned}
i \hbar \partial_{t}\left|\psi_{t}^{\prime}\right\rangle & =\widehat{H}\left|\psi_{t}^{\prime}\right\rangle \text { for } t \in\left[t_{k}, t_{k+1}\right) \quad \text { 'forward' evolution } \\
-i \hbar \partial_{t}\left\langle\psi_{t}^{\prime \prime}\right| & =\widehat{H}\left\langle\psi_{t}^{\prime \prime}\right| \text { for } t \in\left(t_{k}, t_{k+1}\right] \quad \text { 'backward' evolution }
\end{aligned}
$$


are respectively associated. In general, however, it is $\left|\psi_{t}^{\prime}\right\rangle_{t=t_{k+1}} \neq\left|\psi_{t_{k+1}}\right\rangle$ and $\left\langle\left.\psi_{t}^{\prime \prime}\right|_{t=t_{k}} \neq\left\langle\psi_{t_{k}}\right|\right.$. This circumstance is named 'quantum jump' or 'quantum discontinuity'. For $t \in\left(t_{k}, t_{k+1}\right)$ it is formally possible to define the mean values $\left\langle\psi_{t}^{\prime}|\widehat{o}| \psi_{t}^{\prime}\right\rangle,\left\langle\psi_{t}^{\prime \prime}|\widehat{o}| \psi_{t}^{\prime \prime}\right\rangle$, but they have not a direct physical meaning as they are not manifested in an event.

Along this alternative route, the Born rule can be introduced as follows. Let $\widehat{A}=\Sigma_{i} a_{i}\left|a_{i}\right\rangle\left\langle a_{i}\right|$ be a physical quantity. Let us imagine $n \rightarrow \infty$ mental copies of the causal connection between two subsequent events (without intermediate events), and impose that these copies differ only for the final event. The final events will all be, by hypothesis, of the kind $\left|a_{i}\right\rangle\left\langle a_{i}\right|$; this is possible in virtue of the definition of $\widehat{A}$ as a physical quantity, which assures the existence of a manifestation $f$ with these images. The final 'average' event will then be:

$$
\frac{\sum_{n \text { mental copies }}\left|a_{i}\right\rangle\left\langle a_{i}\right|}{n} \rightarrow \sum_{i} P_{i}\left|a_{i}\right\rangle\left\langle a_{i}\right|=\Lambda
$$

From (8) we have $\sum_{i} P_{i}=1$, so that $P_{i}$ is the probability of the manifestation of the $\left|a_{i}\right\rangle\left\langle a_{i}\right|$ event whose existence is guaranteed by the postulate 1 . Since the initial event is fixed, the quantum forward amplitude at the instant immediately preceding the manifestation of the final event is also fixed and we denote it as:

$$
|\psi\rangle=\Sigma_{i} c_{i}\left|a_{i}\right\rangle
$$

Thus, the density operator $\rho=|\psi\rangle\langle\psi|$ associated with this amplitude is also fixed. The operator $\left|a_{k}\right\rangle\left\langle a_{k}\right|$ associated with the final event transforms $|\psi\rangle$ in $c_{k}\left|a_{k}\right\rangle$, and then $\rho$ in $c_{k}^{*} c_{k}\left|a_{k}\right\rangle\left\langle a_{k}\right|$. The same operator transforms $\Lambda$ in $P_{k}\left|a_{k}\right\rangle\left\langle a_{k}\right|$.

The coefficients $c_{i}$ in Equation (9) evolve in time according to Equation (6) but their physical meaning remains undefined. We define it by imposing that the effects of the two transformations (of $\rho$ and $\Lambda$ ) induced by the final event are equal, so that this event induces the transition $\rho \rightarrow \Lambda$. As a result of this definition we obtain $P_{k}=c_{k}^{*} c_{k}$ that is the Born rule.

The postulate 2 can therefore be replaced by the evolution Equation (6) for the forward amplitude, adding as a new postulate the transition $\rho \rightarrow \Lambda$ induced by the final event (projection postulate). It is important to note that, despite the apparent irreversibility implied by both the choice of a determined temporal direction and the decoherence implicit in the projection postulate, such construction is completely time-symmetrical. One could start from the evolution Equation (7) for the backward amplitude, keep the final event fixed and assume the initial event as a variable. In this case the density operator $\rho$ will be the one associated with the backward amplitude at the moment of the initial event, while $\Lambda$ will be the 'initial average event'. One will then have to impose that, as a result of the initial event, $\rho \rightarrow \Lambda$. Postulate 2 seems preferable because it avoids a separate postulate of projection. Before closing this section we would like to discuss briefly two valid concepts both for a manifestly time-symmetrical description and an apparently time-oriented formulation. Let us consider the event $\left|\psi_{t_{k}}\right\rangle\left\langle\psi_{t_{k}}\right|$. If

$$
\left|\psi_{t_{k}}\right\rangle=\sum_{i=1}^{n} \lambda_{i}\left|\phi_{i}\right\rangle, \quad \lambda_{i} \in \mathbb{C}
$$

then the diagonal elements $\left|\phi_{i}\right\rangle\left\langle\phi_{i}\right|$ in the expansion of $\left|\psi_{t_{k}}\right\rangle\left\langle\psi_{t_{k}}\right|$ are named 'virtual sub-events' of the event $\left|\psi_{t_{k}}\right\rangle\left\langle\psi_{t_{k}}\right|$. Crossing one of the two slits in the double slit experiment is a typical example of virtual sub-event. The virtual sub-events are not manifested, that is no application $f^{\prime}: t_{k} \rightarrow\left|\phi_{i}\right\rangle\left\langle\phi_{i}\right|$ exists, as the application $f: t_{k} \rightarrow\left|\psi_{t_{k}}\right\rangle\left\langle\psi_{t_{k}}\right|$ is unique.

The second relevant point is that while the event $\left|\psi_{t}\right\rangle\left\langle\psi_{t}\right|$ represents the localization of the 'quality' $\psi_{t}$ (and the physical quantities associated with it) in the time domain, as for the spatial localization some additional remarks are necessary. The three-dimensional space enters our scheme only through the form of the Hamiltonian $\widehat{H}$. For example, posing: 


$$
\widehat{H}=-\frac{\hbar^{2}}{2 m} \Delta
$$

where $\Delta$ is the Laplacian operator on the Euclidean three-dimensional space $E^{3}$, the equation $i \hbar \partial_{t}\left|\psi_{t}\right\rangle=\widehat{H}\left|\psi_{t}\right\rangle$ admits solutions dependent on $x \in E^{3}$ :

$$
\left|\psi_{t}\right\rangle=\sum_{x \in E^{3}}|x\rangle\left\langle x \mid \psi_{t}\right\rangle=\sum_{x \in E^{3}}|x\rangle \psi(x, t)
$$

The operator impulse is then defined as $\hat{p}=-i \hbar \hat{\nabla}$, and it acts on the space of the solutions $\psi(x, t)$. Equation (12) clearly shows that the operators $|x\rangle\langle x|$ are 'virtual sub-events' of the event $\left|\psi_{t}\right\rangle\left\langle\psi_{t}\right|$. This fact is generally denoted as 'spatial delocalization' of the wave-function $\psi(x, t)$, although an 'a-spatiality' is really involved here, in the sense that the 'position' is not actually manifested except in the particular case of a quality $\psi_{t}$ coincident with a particular spatial position $x$.

It seems useful to point out here that the use of a Hamiltonian operator does not indicate the motion of anything, but rather it has to be seen as a 'probability gradient', a notion that unifies different formalisms such as Bohm potential and Feynman path integrals [22]. The fact that the application $f$ introduced with the Postulate 1 is "sensitive" to time but not to space generates the well-known phenomenon of the non-separability of entangled amplitudes, well exemplified by the amplitudes of a pair of identical particles of spin $1 / 2$ in a state of singlet. If one of the two particles is sent to a polarizer that separates the two beams with opposite spin values, each of which is subsequently directed to a detector, it is possible to have a "click" in one of the two detectors. This means that at that moment $f$ localizes a precise spin value (better, a value of the spin component along the measurement axis) in a spatial region corresponding to the detector volume. With this, the virtual sub-event corresponding to the specific component of the singlet actually selected by the measurement becomes a real event. The spin of the other particle is therefore localized in time at the same instant. It remains not localized in space, but it can become so if the other particle is also subjected to a similar measurement.

\section{General Remarks on Measurements}

Temporal localization is associated with interaction micro-events (quantum jumps) and not with measurements performed by an experimenter. The measurement procedures were therefore absent in our previous discussion. It is only now that, having defined the context of a more fundamental quantum reality whose events are temporal localizations, we can move on to the description of measurement procedures as particular physical processes within that reality. These procedures involve particular macroscopic entities called "measurement apparatuses"; we must therefore define, in succession, classical macroscopic bodies and measurement apparatuses in the context of the quantum reality constituted by temporal localizations. We emphasize that this is a fundamental difference with respect to the conventional formulation, which is notoriously agnostic about the existence of a quantum reality. We call 'classical macroscopic body' a cluster of events whose averaged properties evolve deterministically over time, within the limits allowed by the finiteness of the quantum of action $h$.

Let us try to clarify formally the concept, at least sufficiently for the purposes of our argument. If the cluster were empty, that is, there were no events but only the possibility of their manifestation, such possibility would be (we assume) expressed by quantum amplitude $|\psi\rangle$, to which the density operator $\rho^{\prime}=|\psi\rangle\langle\psi|$ would correspond. We now postulate that $|\psi\rangle$ is decomposable in a basis of amplitudes $\left|\psi_{k}\right\rangle$, such that the actual manifestation of the events inside the cluster reduces $\rho^{\prime}$ to its diagonal component in this basis. In other terms, $\rho^{\prime}$ becomes a linear superposition $\rho$ of density operators $\rho_{k}=\left|\psi_{k}\right\rangle\left\langle\psi_{k}\right|$ :

$$
\rho=\Sigma_{k} \lambda_{k}^{*} \lambda_{k} \rho_{k} ; \quad \Sigma_{k} \lambda_{k}^{*} \lambda_{k}=1 ; \quad \lambda_{k}=\left\langle\psi_{k} \mid \psi\right\rangle
$$


When this happens we will say that the cluster is a classic macroscopic body. In practice, (13) expresses the total decoherence of $\rho$ in a basis selected by the dynamics of the events themselves.

Of course, the quantum equation of time evolution (whose validity is here assumed as universal) applies to the operator $\rho^{\prime}$, and it can be objected that in general this evolution does not lead to the final 'state' (13). This objection, however, does not take into account that the quantum evolution of the amplitudes (or more generally of the density operator), as it is usually defined, is relative to the unperturbed situation between two successive events. It defines the probability of occurrence of the next event, but the actual manifestation of this one modifies the initial condition of the next evolution stage. Consequently, the quantum equation of time evolution must be applied starting from this new condition and this happens many times in a single unit of time. The unitarity of the time evolution is thus broken and the average result is decoherence. A classical macroscopic body is made decoherent by the same discontinuities that constitute its essence, in a basis depending on its internal dynamics.

According to this view, a classical macroscopic body is an object, which is actualized in space and time precisely because it is a complex of actualizations in the temporal domain (events). It is persistent over time and endowed with a substance (its events) with attributes (the average properties of the cluster). The evolution of these attributes can admit a classical (approximate) description. On the other hand, the single event and the connection between two successive events represent phenomena that cannot be classically described and therefore are, in this sense, 'entirely quantum'.

Let us consider now the elements $\left|\phi_{k}\right\rangle$ of a second Hilbert space $\mathbf{H}$ (the "particle"); we assume these elements are the eigenvectors of a physical quantity $\widehat{o}$. If the manifestation of the event $\left|\phi_{k^{\prime}}\right\rangle\left\langle\phi_{k^{\prime}}\right|$ at the instant $t_{e v}$ implies with certainty the transition $\lambda_{k} \rightarrow \delta_{k k^{\prime}}$ for any value of $k, k^{\prime}$ and $t_{e v}$, this transition is called a 'measurement' of $\widehat{o}$, with 'result' $\left|\phi_{k^{\prime}}\right\rangle\left\langle\phi_{k^{\prime}}\right|$. The classical macroscopic body is then called a 'measurement apparatus' of the physical quantity $\widehat{o}$.

Denoting with $|\phi\rangle=\Sigma_{k} c_{k}\left|\phi_{k}\right\rangle$ the probability amplitude of the events $\left|\phi_{k}\right\rangle\left\langle\phi_{k}\right|$, evaluated at the instant $t$, the density operator associated with the complex measurement apparatus plus particle is then defined as $\rho|\phi\rangle\langle\phi|$ where $\rho$ is defined by relation (13). Since the interaction between the particle and the apparatus, mediated by the QJ, is diagonal on the basis $\left|\psi_{k}\right\rangle\left|\phi_{k}\right\rangle$, this operator evolves to:

$$
\rho_{c}=\Sigma_{k} c_{k}^{*} c_{k} \lambda_{k}^{*} \lambda_{k} \rho_{k}\left|\phi_{k}\right\rangle\left\langle\phi_{k}\right|
$$

This expression equates the diagonal component (on the basis $\left|\psi_{k}\right\rangle\left|\phi_{k}\right\rangle$ ) of the density operator calculated in absence of quantum jumps, that is $\rho^{\prime}|\phi\rangle\langle\phi|$, to which the same consideration previously made for $\rho^{\prime}$ can be now applied. Measurements are made possible by selective coupling between the 'particle' operators $\left|\phi_{k}\right\rangle\left\langle\phi_{k}\right|$ and the (already) de-coherent $\rho_{k}$ states of a classical macroscopic body. Before the quantum jump, each $\rho_{k}$ is coupled to any $\left|\phi_{k}\right\rangle\left\langle\phi_{k^{\prime}}\right|$ and vice versa (without entanglement); the quantum jump selects a specific coupling $\rho_{k}\left|\phi_{k}\right\rangle\left\langle\phi_{k}\right|$.

We deal with a two stage measurement process. The first stage involves only microscopic components of the measurement apparatus and consists of the interaction that determines the manifestation of the event $\left|\phi_{k^{\prime}}\right\rangle\left\langle\phi_{k^{\prime}}\right|$. The second stage consists of amplification/registration of this event, which in turn determines the transition $\lambda_{k} \rightarrow \delta_{k k^{\prime}}$. While the amplification/recording phenomena can be described, with high precision, in classical language (which does not preclude their exact description in quantum terms), the first stage is entirely quantum. The manifestation of $\left|\phi_{k^{\prime}}\right\rangle\left\langle\phi_{k^{\prime}}\right|$, when considered in itself without regard of the following amplification/recording, rather represents the localization of the value $o_{k^{\prime}}$ of $\widehat{o}$ in the time domain. It is our opinion that these localizations correspond to what other researchers have called 'hidden measurements' $[23,24]$. Several non-local or contextual ' $\varepsilon$-machines' can be imagined. For example, in $[25,26]$ the Born rule is exactly reproduced by the selection of a specific 'loop' associated with a specific 'transaction'. However, these aspects are beyond the scope of this paper and we leave them open. As it can be seen, with respect to standard presentation the difference lies mainly in the differentiation that is made between events (quantum jumps), which are localizations of packets of physical quantities in time domain, and measurement procedures. To clarify further the concept, let us examine two concrete cases. Let us first imagine an 
electron incident on a single slit screen. If the electron is absorbed by the screen, a quantum jump occurs in the Hilbert space associated with the combination 'electron + atom of the screen absorbing the electron'. In this jump, the quantum amplitudes that represented the electron in flight and the atom in a stationary state are transformed into new amplitudes describing the electron bound to the atom and this one (we say) in an excited orbital. This quantum jump takes place at a precise instant of time, corresponding to the absorption of the electron; it localizes both the electron and the atom in time domain. At the same time, the electron is localized in the three-dimensional space with an accuracy defined by the volume occupied by the final atomic orbital. If the electron is not absorbed and passes through the slit (negative interaction with the screen), it is only the quantum amplitude of the electron to undergo a quantum jump at the time of passage. This jump converts the quantum amplitude of the electron into a new amplitude that exhibits the phenomenon of diffraction. It localizes the electron in time (moment of passage) and at the same time in three-dimensional space; the accuracy of spatial localization is defined by the slit size. In these processes an interaction occurs (real in one case, negative in the other) with temporal localization of a packet of physical quantities. Such localization is the quantum jump. However, the localization event is not amplified (nor recorded) on a macroscopic scale and there is therefore no measurement. The second case is that of an electron which impacts a photographic plate and reduces a silver atom contained within a silver halide granule dispersed in the plate emulsion. This case is quite similar to that of the real interaction with the screen atom, with the difference, however, that the microscopic reduction event can be amplified macroscopically through the photographic process (development, fixing, washing). During this process, the state of all the atoms of the granule is at first changed (amplification); this change is successively made permanent (recording) through the fixing process. The final result is a macroscopic modification: the appearance of a darkened granule in the plate emulsion. The subsequent scanning of the plate can only detect an already existing condition: the presence of a darkened grain.

The following inferences seem therefore clear: (1) the measurement process is a possible, but not necessary, concomitance of the electron temporal localization event and these two things should not be confused; (2) before the quantum jump, the electron is spatially delocalized in correspondence of the positions of a multitude of silver atoms in different grains and each grain interacts with the entire electron; (3) when the jump occurs, this delocalization is reduced and the electron is spatially localized in correspondence of a specific silver atom within a single, specific grain; (4) before its interaction with the electron, and regardless of it, each grain was actualized as a multitude of quantum jumps; the interaction simply modifies the course of this actualization. Therefore, no Schrödinger cat situation arises. This situation is modeled by Equation (14) as follows: $\lambda_{k}^{*} \lambda_{k}$ represents the fraction of silver atoms in $k$-th grain; $c_{k}^{*} c_{k}$ is the probability of presence of the electron in correspondence of the $k$-th grain. The quantum jump selects a specific state $\rho_{k}\left|\phi_{k}\right\rangle\left\langle\phi_{k}\right|$ with probability $\lambda_{k}^{*} \lambda_{k} c_{k}^{*} c_{k}$.

The main advantage of this proposal lies, in our view, in the most definite delimitation of the role of measuring apparatus. To illustrate the concept, consider the distribution of molecular speeds in a gas at a definite temperature. The properties of this distribution are determined by the impacts between the gas molecules and between these and the molecules of the vessel walls. These impacts are all QJs that localize molecules in space-time. However, none of these impacts, which contributes to defining the classical system 'gas', is observed by the experimenter. In fact, the amplification and recording of the single impact is lacking (the system is at the equilibrium and without memory) so that we cannot speak of the energy and impulse exchanged in these impacts as 'observables'. It is instead appropriate to talk about objective physical quantities associated with the single molecule or exchanged in the single impact.

Thus, it is justified the well-known fact that quantum mechanics can be successfully applied to phenomena that are not actually observed, such as the formation of a single chemical bond in a bulk of molecules or the impacts of gas molecules with the walls. Experimental apparatuses represent a condition in the definition of quantum amplitudes, but they are not their cause. Thus, even the known 
difficulties encountered in the application of the formalism to cosmology are removed, especially in the first moments after the initial singularity, when no observer and no setup could still exist.

\section{Considerations about the "Cat Paradox"}

In this section we apply the formalism defined in the previous sections to the concrete case of the pointer states of a measurement apparatus interacting with a micro-entity. In particular, we will consider the paradigmatic case of the "cat paradox" [27], trying to highlight how this paradox does not occur in the present formulation. In the conventional version of the QM the "cat paradox" is represented by the following situation:

$$
A|G\rangle|D\rangle+B|E\rangle|L\rangle
$$

where: $G=$ nucleus in ground state, $E=$ nucleus in excited state, $L=$ live cat, $D=$ dead cat. Let us see now how things are in the formulation we propose. First, the two states of the cat are decoherent because they are associated with distinct sets of actualizations (micro-events). Each of them is coupled with a distinct value of a dichotomous variable: "the QJ occurred" or "the QJ did not occur". The QJ is here the nuclear decay. Consider the nuclear amplitude:

$$
\psi_{n}=A|G\rangle+B|E\rangle
$$

The occurrence of the QJ corresponds to the action of the projector $|G\rangle\langle G|$ on $\psi_{n}$; the result of this action is $A|G\rangle$. The non-occurrence of the $Q J$ corresponds to the action of the operator $1-|G\rangle\langle G|$ on $\psi_{n}$; the result of this action is $B|E\rangle$. We have therefore the two decoherent couplings $A|G\rangle|D\rangle$ and $B|E\rangle|L\rangle$ that originate the total density matrix:

$$
\Omega=A A^{*}(|G\rangle\langle G|)(|D\rangle\langle D|)+B B^{*}(|E\rangle\langle E|)(|L\rangle\langle L|)
$$

which is the particular form assumed by Equation (14) for the specific problem. The single experimental run begins with the preparation $(|E\rangle\langle E|)(|L\rangle\langle L|)$, corresponding to the fact that a $Q \mathrm{~J}$ has not yet occurred. The nuclear amplitude evolves as a superposition of $G$ and $E$ until the decay occurs. When the decay occurs, the density matrix relative to the single run becomes $(|G\rangle\langle G|)(|D\rangle\langle D|)$. This sudden transition is the killing of the cat. The unitary time evolution of the superposition of $G$ and $E$ has no reflection on the status of the cat in the single run, except when the QJ occurs. What happens is that the QJ converts the qubit $\psi_{n}$ to the corresponding bit " $E$ or $G$ ". After that the macroscopic measurement apparatus couples with this bit. Thus the QJ transforms quantum information in classical information.

The decoherence of the $L, D$ states of the cat makes them refractory to the superposition, and therefore unsuitable to act as the basis states of a qubit that can be the input of a QJ. They can only read the occurrence (or non-occurrence) of the QJ. Instead, the states of the particles that contribute in a vertex of interaction to produce a QJ can undergo to a superposition and are in fact, generally, entangled. The unitary time evolution concerns the qubit before the QJ; it affects the apparatus only through the conversion of the qubit by the $Q J$. The time evolution of $\Omega$ is then non unitary.

In the conventional version, quantum amplitudes (bra or ket) are associated with the states of a system. It turns out therefore incomprehensible that the same physical situation (the cat + nucleus complex) is simultaneously represented by a superposition and a mixture. Two "states" cannot in fact be superposed and inchoerent at the same time. In this version, however, the amplitudes are associated with events and not with states of any system; moreover, the nature of this association is different for the superposition and for the mixture.

In the conceptual experiment of the cat, the superposition of amplitudes associated with the nucleus (the "qubit") is relative to the two outcomes $G$ and $E$, conditioned by past events (the "preparation"), of an event that has not yet occurred. The conjugated superposition is relative to the 
results, conditioned by future events (the "detection"), of a past event already happened. The elements of the density matrix of the cat + nucleus complex (the "bit") are instead related to events actualized in the present moment and to their logical negatives: the single nucleus is "already decayed" $(G)$ or "not-yet-decayed" (E).

As can be seen, the essential point is that the cat is a set of actualizations and its qualifications $L$ and $D$ represent two distinct complexes of classical properties $a, b, \ldots z$ defined on this set, in a way such that:

$$
L=a \wedge b \wedge c \ldots \wedge z ; \quad D=(\neg a) \vee(\neg b) \vee(\neg c) \ldots \vee(\neg z) \Longrightarrow L=\neg D
$$

Moreover:

$$
x \wedge(y \vee z)=(x \wedge y) \vee(x \wedge z) ; \quad x, y, z=a, b, \ldots, z .
$$

In this expressions the symbols $\neg, \vee, \wedge$ represent respectively the negation, the inclusive disjunction (the Latin vel) and the conjunction of classical logic. We immediately see that the properties attributed to the cat do not respect the relations of quantum logic. The distributivity of $\wedge$ with respect to $\vee$ means that properties $a, \ldots, z$ are relative to a level of description that is enormously coarse if compared to the fineness of the quantum of action; on this level the possible non-commutativity of physical quantities does not play any role. A property of this type could be, for example, "inside the cat the blood circulation is active" or the opposite. It is evident that, with respect to the truth of statements of this kind, the quantum delocalization is irrelevant and we are therefore in the full domain of application of classical physics. Let us thus consider the quantum amplitude:

$$
\psi=\alpha|D\rangle+\beta|L\rangle ; \quad \alpha, \beta \in \mathbb{C} ; \quad|\alpha|^{2}+|\beta|^{2}=1 .
$$

The projector $|\psi\rangle\langle\psi|$ describes a set of classical properties, as requested, if and only if $|\alpha|^{2}=1$ or $|\beta|^{2}=1$. Otherwise, although it is a well-formed expression of quantum formalism, it will have an empty semantic set. It is the same situation that occurs in the grammar of the ordinary language with expressions like "the liquid pencil" or "the children of a sterile woman". In terms of the axiomatic discussed in this paper, $|\psi\rangle\langle\psi|$ can be manifested as an event if and only if $|\alpha|^{2}=1$ or $|\beta|^{2}=1$ :

$$
(\exists t \in T, f: t \rightarrow|\psi\rangle\langle\psi|) \Longrightarrow\left(|\alpha|^{2}=1\right) \vee\left(|\beta|^{2}=1\right)
$$

Accordingly, in our re-formulation of quantum mechanics, the measurement apparatus is not in a superposition of pointer states. Therefore, if the measurement apparatus and the micro-entity undergoing the measurement are closed inside a box inaccessible to an external observer, who however knows the initial state of the complex apparatus + micro-entity, this observer cannot legitimately deduce that such complex is in a superposition state. In fact, this is not the law of evolution of this complex in our version of quantum mechanics. Therefore, paradoxes such as that of "Wigner's friend" cannot arise, nor more elaborate paradoxes such as the one recently discussed by Frauchiger and Renner $[28,29]$.

\section{Quantum Jumps and Bits: Localization in Time as Information}

In this section, in which we will take the liberty to be a little more speculative, we would like to reconsider the idea of 'event', understood as 'localization in time', from an informational perspective. It seems to us that a possible starting point in this direction is represented by the uncertainty principle. In our proposal, this fundamental principle of QM describes the intrinsic limitation of the manifestation of physical quantities on a quantum scale (let us consider, for example, the a-spatiality already mentioned in the case of the position). An expression of type $\sigma(q) \sigma(p) \geq h / 4 \pi$ indicates that it is not possible to reduce the product of the amounts of delocalization $\sigma(q)$ of the position $q$ and $\sigma(p)$ of the momentum $p$ below the limit value $h$; this value then sizes the volume of an elementary cell in the phase space. The volume occupied by a physical system in its phase space therefore contains a finite number of distinguishable states. The information associated with the manifestation of one of 
these states is therefore finite, though it may be enormous. Bekenstein estimated an upper bound for information $I$ associated with a system with total energy $E$ enclosed in a sphere of radius $R$ in ordinary three-dimensional space [30]:

$$
I=\log _{2} n \leq \frac{2 \pi E R}{\hbar c \ln 2}
$$

The finiteness of $I$ defines a range of classical values attributable to two non-commuting variables such as position and momentum. An examination of the system on a finer scale leads us into realms dominated by delocalization and entanglement, such as that of atomic orbitals and their transitions [31]. On the other hand, a compromise between classical properties and quantum uncertainty can arise when weak measurements are performed [32].

A particular form of the Bekenstein constraint valid for confined systems within a horizon of events (it was originally deduced for black holes in [33]) is the following:

$$
I=\frac{A}{4 l^{2}}
$$

where $A$ is the horizon area and $l \approx 10^{-33} \mathrm{~cm}$ is the Planck length. $I$ represents the information enclosed within the horizon and therefore 'lost' from the point of view of the world outside the horizon, for which it represents the entropy associated with the horizon.

Let us consider now the transition of a physical quantity, delocalized in time, to its condition completely localized in time, the passage we identified with the notion of 'event' or QJ. Such a passage corresponds to the acquisition of information on the temporal localization of the physical quantity (or the packet of physical quantities). If we compare with a metaphor inspired by information technology, the time domain to a memory storage device (we say a hard disk), then the 'event' is the irreversible act of writing a data packet on the disk. In systemic terms, we can talk about the informational openness of QM, correlated with the conversion of primordial non-local information into a measurable form according to Shannon and Turing. Primordial information is clearly a formal cause in the Aristotelian sense, with the additional characteristic of being synchronic and therefore very different from the diachronic efficient cause which is usual in physics (dynamical causality expressed by the unitary evolution of amplitudes). This one is probably only an appearance of this deeper formal causation, as perceived from the time domain. Bohm proposed the term 'active information' [34]. There are very interesting models on these aspects, such as the implicate order explored with non-commutative geometry by Basil Hiley, but here we will not go into details on this $[35,36]$. All this suggests that the natural habitat of QM is pre-temporal [37,38].

One can ask if there is no minimum proper time interval $\theta_{0}$ between two successive temporal localizations of the same particle. One can also wonder whether the coordination of events by an observer is not limited within a horizon of radius $c t_{0}$ centered on the observer itself $(c$ is the limit speed). We can attempt to estimate $t_{0}$ from the experimental value of the cosmological constant $\lambda$, which is in the order of $10^{-56} \mathrm{~cm}^{-2}$. Assuming that the origin of such a constant is the presence of a de Sitter horizon, the relation $\lambda=\frac{4}{3 c^{2} t_{0}^{2}}$ must hold, which provides $c t_{0} \approx 10^{28} \mathrm{~cm}$. The de Sitter horizon area is then $A \approx\left(10^{28} \mathrm{~cm}\right)^{2}$ and inserting this value into (23) we obtain $I \approx 10^{123}$.

We can divide the portion of the contemporaneousness space of the observer, internal to the observer de Sitter horizon, in 'cells' of volume $\left(c \theta_{0}\right)^{3}$, each corresponding to a distinguishable spatial localization. Since the radius of the horizon is $c t_{0}$, the number of such cells is $N \approx\left[\left(c t_{0}\right)^{3} /\left(c \theta_{0}\right)^{3}\right]$. Each cell can be in one of two states: 'on' if a localization occurs in it, 'off' otherwise. The number of possible states is clearly $2^{N}$ and the information associated with these states is $\log _{2}\left(2^{N}\right)=N$. We assume that quantum jumps manifest the elementary components of the physical system within an elemental cell of volume $h^{3}$ in their phase space, so that each state will correspond to a cell of volume $h^{3 N}$ in the phase space of the total system. The logarithm of the number of states is therefore the same information $I$ of $(23)$ and we have $I \approx\left[\left(c t_{0}\right)^{3} /\left(c \theta_{0}\right)^{3}\right]$. From this relation we obtain $c \theta_{0} \approx 10^{-13} \mathrm{~cm}$, a result that is of the same order of both the classical radius of the electron and the range of strong 
interactions (color confinement). The time interval $\theta_{0} \approx 10^{-23} \mathrm{~s}$ is of the same order of the 'chronon' introduced by Caldirola in his classical theory of the electron, just as the time interval between two successive localizations of the electron in space-time [39,40].

If this reasoning is correct, the impact of the finiteness of $I$ on the temporal localization process would consist in simultaneous appearance of two scales, both independent on cosmic time: one cosmological (de Sitter radius), the other at the particle level (chronon). This suggestion could open new perspectives of unification between elementary particle physics and cosmology.

We also observe that, starting from the two fundamental constants of the localization process, i.e., the intervals $t_{0}$ and $\theta_{0}$, it is possible to define a maximum acceleration $c t_{0} /\left(\theta_{0}\right)^{2}$. On a spatial interval $c \theta_{0}$, corresponding to the typical scale of the elementary particles, this acceleration is reached if a speed variation $c \theta_{0} /\left(d_{P} / c\right)$ is implemented in a temporal interval $d_{P} / c$, where $c t_{0} /\left(\theta_{0}\right)^{2}=$ $c \theta_{0} /\left(d_{P} / c\right)^{2}$. Substituting $I=\left[\left(c t_{0}\right) /\left(c \theta_{0}\right)\right]^{3}$ and $A=4 \pi\left(c t_{0}\right)^{2}$ in Equation (23), we can see that $d_{P} \approx l$. It is of course possible to proceed in reverse order, defining the Planck scale through the maximum acceleration, and thus obtain the (23). The foundation of (23) therefore appears to be the global-local connection manifested in the process of localization, rather than some form of "holographic principle". This suggests a purely informational interpretation of the Planck scale. The connection of this scale with the universal gravitational constant could be accidental and perhaps due to the limitations imposed on the principle of local equivalence between gravity and inertia by the existence of a maximal acceleration. We leave this topic open for subsequent research work.

\section{Quantum Computing in and Beyond Spacetime}

In this section we will discuss the problem of the possible existence of spatiotemporal constraints on the quantum computing, raised by some authors [20,21]; our reference context will be the one described in the previous section. We will argue that such a constraint exists on the cosmological scale, but that it is in practice unattainable and, in any case, devoid of effects on the actually implementable quantum computing schemes. Our reasoning can be considered an answer to Davies [20]. We will start by considering the case of a single qubit:

$$
\psi=\alpha|+\rangle+\beta|-\rangle
$$

This qubit will be by hypothesis associated with a particle. We also assume that the basic amplitudes $|+\rangle,|-\rangle$ are spatially encoded, in the sense that the attribution of one of these two amplitudes to the particle corresponds to its localization in one of two distinct spatial regions. We can consider the actual case of a particle of spin $1 / 2$ sent to a Stern-Gerlach analyzer which separates the two components of spin along the direction of the applied magnetic field. The click of the counter " + " downstream of the analyzer will be at the same time the measurement of the spin with result $+1 / 2$ and the spatial localization of the particle within the volume of the counter. A similar consideration will apply to the click of the " -" counter. Consider then a system of $n$ particles, each associated with a qubit with basis amplitudes distinct from those of all the others. By encoding each of these amplitudes with a distinct spatial region, the total amplitude of the $n$ particles will be a superposition of $2^{n} n$-ples of distinct spatial regions. While the quantum computing occurs at the level of the phase relations between these $n$-ples, which are a-spatial and therefore not subject to any constraint of spatial nature, the situation is a little different for the single $n$-ple. It is indeed evident that every $n$-ple of space regions must belong to space, and therefore be contained in it as a subset. Now, the maximum number $n$ of qubits with spatially codable basis amplitudes will be given by the maximum number of possible spatial positions for a particle at a given moment, within the cosmological horizon. As we have argued in the previous section, this number is $I \approx 10^{123}$. In other words, the number of qubits will be subject to the Bekenstein limit $n \leq 10^{123}$. Naturally this limit is satisfied by all current and future quantum computer projects. However, it is also possible to show that it is unreachable by computers made up of ordinary matter with stable nuclei and electrons. The average density of ordinary matter in the 
Universe (with the exclusion of dark matter and dark energy) is $\approx 5 \times 10^{-31} \mathrm{~g} / \mathrm{cm}^{3}$. Almost all of the mass is represented by nucleons, with an individual mass of $1.67 \times 10^{-24} \mathrm{~g}$. It is obtained hence a nucleonic density of $3 \times 10^{-7} / \mathrm{cm}^{3}$. About half of the nucleons consists of protons and assuming that their charge is neutralized by as many electrons, an electronic density of $1.5 \times 10^{-7} / \mathrm{cm}^{3}$ is obtained. We have therefore a particle density of $4.5 \times 10^{-7} / \mathrm{cm}^{3}$. Assuming a de Sitter radius of $1.4 \times 10^{28} \mathrm{~cm}$, there are therefore in total $0.5 \times 10^{79}$ particles (nucleons and electrons). A lot less than $10^{123}$.

\section{Comparisons with Other Approaches}

No theory is born of nothingness, and every scholar is well aware of walking with others. In this section we aim to take stock of the kinships that have been a source of inspiration for us. Although the formal tools introduced in this work are the same as the current formulation of the $\mathrm{QM}$, the prescriptions on their use are different, and this leads to a difference in the predictions obtainable with the two versions of the theory. Naturally, nothing new can be expected regarding the description of what in the usual jargon can be defined as "micro-systems" represented by "pure states"; therefore, the representation of the configuration in isolation of atoms, atomic nuclei and molecules-for example-will be the same. However, there will be significant differences in the treatment of interacting systems, in particular those with many particles, since they will present, in appropriate limit conditions, transition phenomena to the status of classical objects not described in the usual version. In our proposal, therefore, the inclusion of a specific ontological element (the identification of the reduction of the amplitude with the physical phenomenon of the "jump") leads to predictive differences not obtainable in the context of a simple interpretation of the theory in its current formulation.

Other approaches try to obtain similar results by modifying the dynamics of the theory, a stratagem that is not used in the context of our proposal. This is the case of spontaneous localizations contemplated by the Ghirardi-Rimini-Weber (GRW) approach, both in the form of "hits" and as continuous processes obtained by adding appropriate stochastic terms to the quantum motion equation [41-44]. In particular, the version known as GRW-flash [45-47] may have some similarities with our proposal, so it is important to summarize also the differences. First of all, we do not hypothesize spontaneous localizations (that is, independent from ordinary interactions) that happen randomly and that remain elusive on the experimental plan. Instead, we identify the processes of reduction of the wave function with specific objective physical phenomena whose existence is today experimentally well demonstrated: the quantum jumps. These jumps are induced by the usual physical interactions (electromagnetic, weak, nuclear etc.).

Our description, therefore, does not contemplate the possibility of spontaneous flashes distinguished from ordinary interactions, but has instead to do with a quantum discontinuity connected with such interactions [31]. This point is relevant with reference to the measurement theory (see the conclusion of [47]). Moreover, we see no reason to favor the positional basis and we mean the localization in a temporal, not a spatial sense. What is instantiated is the projector on the quantum amplitude and not the spatial position. We see the peculiarity of the quantum description in the fact that, according to it, the ordinary interactions modify the amplitudes according to one or the other of two causal schemes that are mutually irreducible and, at the same instant of time, mutually exclusive: the unitary evolution and the QJ. To the dynamical causation (which involves the transfer of energy between spatio-temporal regions) a formal causation is added (which involves a certain localization information entering the spatio-temporal domain).

The appearance of a particle scale in the context of the basic formalism recalls the representation of "corpuscles" as localized concentrations of field energy, guided by a pilot wave; a representation typical of non-linear theories such as those proposed by the de Broglie school (see e.g., [48,49]). The difference, however, is that in the context of the present approach (which preserves the linearity of conventional formalism) we describe the temporal localization of physical quantities, rather than the spatial localization of a pre-existent globule. In other words, our idea of objectivity of the physical 
world mirrors an ontology of events rather than substances, according to Russell and Whitehead's classic ideas.

In describing both the relata and the relations, i.e., quantum jumps and transition amplitudes respectively, we have used their full temporal symmetry offered by the QM in its usual version. We have therefore considered both the transition amplitude and its conjugate and, for the QJ, both the ket and its conjugated bra. Both these choices recall the Transactional Interpretation of the QM (TIQM) introduced by Cramer in the 1980s [50-52] and advocated in particular by Kastner (see eg [53] for a general presentation of her views). As far as we are concerned, we have carefully considered the TIQM since its appearance, letting us be inspired by its principles. However, we have developed these principles in a different form from the one originally proposed by Cramer [26,54]. Our personal elaboration naturally connects to the postulates presented in this article and clarifies them. However, it has led us on a path that differs from the TIQM for some significant differences, which we will now try to summarize.

The basis of transactional narrative is the simultaneous emission of the "offer" and its conjugated (the "confirmation"), respectively in the future and past light cone of the emission point. The same status of reality is attributed to the offer and the confirmation and the superposition of offers and confirmations induces the exchange of quanta. The emitter is therefore also an absorber, and the whole description is traced back to specific properties of the absorbers [55]. It seems to us that this description concerns the propagation of specific fields on the temporal (or spatio-temporal) domain and is therefore internal to this domain. It involves, even in the process of double emission or absorption-emission, the only efficient diachronic causality; that is, it has to do with a dynamical causality in space-time. In fact the reference model assumed by TIQM is the Wheeler-Feynman electrodynamics [50].

The basis of our reformulation is instead the localization of physical quantities in the temporal domain. The causality involved in the localization process is formal, not efficient; it is synchronic (in that it connects the atemporal realm of quantities, that is, Hilbert space, to temporal domain), rather than diachronic (connection between instants). There is no process of emission or absorption of quantum fields in the classical sense of the term. There is no object, field or particle, which acts as emitter-absorber. The connection between events is timeless (the time labels the different actual localizations) and the transition amplitudes are a time-symmetrical aspect of this connection: there is no propagation of fields in time or space-time. The role of the offer-confirmation pair is assumed here by a projector, that is, an algebraic entity that represents a transformation, and which can be matched with the elements of a classical propositional calculus. To be more exact, we consider a cosmic process of manifestation that associates a temporal label to the projectors, thus carrying out a localization action to which information is associated, which is thus "entered" in the temporal domain; and in this sense we speak of a formal cause. The process we are trying to describe seems to be more fundamental than what can be captured with the ontological categories of a classical field theory, even if extended in a time-symmetric and non-local sense and applied to quantum fields. It is sufficient to consider that the potential non-separability of amplitudes appears here on a native level.

In this sense, perhaps the closest suggestion is the implicate order of Bohm-Hiley [56], however, with the important clarification that its explication occurs at the level of quantum discontinuity. As we have seen, informational considerations on discontinuity seem to connect in a natural way to two important scales: that of elementary particles and that of Planck, perhaps opening up new perspectives of foundational research on a theory of inestimable success like the QM.

Another research program with which our approach has clear convergences is that of decoherence [57-62]. Therefore, it seems opportune to emphasize here the similarities and differences, and we think that the best way to do this is to discuss briefly an elementary ideal case. Consider a classical macroscopic system; as we have seen, it consists of a normally very large number of elementary quantum components (for example, molecules). Let $S$ be one of these components and $E$ the complex consisting of the remaining components. Let us consider a specific interaction between $S$ and the individual elements of $E$ such that the total amplitude of $S+E$ at time $t$ is: 


$$
|\psi\rangle=\alpha\left|s_{1}\right\rangle\left|e_{1}\right\rangle+\beta\left|s_{2}\right\rangle\left|e_{2}\right\rangle
$$

For simplicity, we have considered only two (orthogonal) basis amplitudes $s_{1}$ and $s_{2}$ of $S$ and two (not necessarily orthogonal) amplitudes $e_{1}$ and $e_{2}$ of $E$. Now consider the trace of $|\psi\rangle\langle\psi|$ on the states of $E$; it will contain terms of interference proportional to the real part of the scalar product of the two states of $E$. If these ones are orthogonal, the interference disappears, whereas in the general case it will be attenuated. Because $S$ is randomly chosen among the elementary components of the macroscopic system, and this random choice determines the components of $E$, we have that the possible existence of an interaction of this type between $S$ and $E$, independent on this choice, leads to a quantum decoherence between the elementary components of the system. This decoherence makes the system, in a certain sense, "more classical" because it diagonalizes the density matrix of each of its components, averaged on the degrees of freedom of the others, on a basis selected by the interaction itself. Of course, coherence does not disappear, it is simply transferred from the basis elements of $S$ to the amplitudes of $S+E$. This is the mechanism underlying the theory of decoherence. In our approach all this remains true. Now, however, at a given instant of time the total amplitude expressed by the preceding equation "collapses" into one of the basis amplitudes of $S\left(s_{1}\right.$ or $\left.s_{2}\right)$ due to a quantum jump. The probability of the two results is defined by the diagonal components of the density matrix in the basis $s_{1}, s_{2}$. After the collapse, the off-diagonal coefficients reappear as a result of unitary evolution, until a subsequent quantum jump. Thus the mean density matrix will have both diagonal and off-diagonal terms. The decay of the off-diagonal terms in a "multi-hit" process (where each "hit" is a QJ) was analyzed by Simonius in a pioneering work [63]. The decoherence time is a function of the frequency of the Rabi oscillations of the free $S$ system, of the degree of orthogonality between the amplitudes of $E$ and of the (normally Poissonian) temporal distribution of "hits", in turn dependent on state variables such as temperature and pressure. The mean effect of a succession of quantum jumps on the evolution of the density matrix therefore seems to be what the theory of decoherence describes. From our point of view, however, the fundamental process is a piecewise unitary evolution, whose intervals are joined by genuinely non-unitary discontinuities.

\section{Conclusions}

As is well known, the absence of trajectories in quantum formalism and the instantaneous cancellation of the wave-function when the particle impacts on an absorber make any interpretation of Quantum Mechanics (QM), based on a realistic classical approach, extremely difficult. This difficulty has generated the two still dominant trends in the debate about the nature of QM. On the one hand, the idea of the incompleteness of the theory goes back to Einstein and arrives to current proposals like that of 't Hooft. On the other hand, the 'pragmatic' approach of Bohr is centered on what the observer can say of the world through measurement procedures (for a good recent review, see [1]). It should be said that none of the two trends provides universally shared answers for the weirdest aspects of QM. We recall that the 'realistic' and 'pragmatic' trends have been established long before the experimental confirmation of non-local aspects. If, for the Copenhagen view, non-locality is an 'unexpected host', for realistic theories it is difficult to reconcile QM and relativity. Both of the interpretative lines have tried to retain the image of persistent micro-physical object bearers of persistent properties. Realists used 'globules' driven by a medium, while pragmatists saw quantum amplitudes as descriptors of the 'state' of a 'system'. Our re-reading of QM basic postulates identifies the 'quantum jump' with the notion of 'physical event', i.e., the temporal localization of a set of physical quantities, without any necessary relation with the measurement processes and apparatus. The causal connections between events is assured by the unitary time evolution of amplitudes, corresponding to a condition of temporal de-localization of those same quantities. This connection (if any) is probabilistic, and is described by the Born rule. No 'micro-object' is assumed in the time interval between two subsequent events. The quantum amplitudes associated with the preparation or post-selection are therefore not 'states' of any 'system'; in particular, a linear superposition of these amplitudes has not 
to be intended as a superposition of 'states'. Temporal localizations of physical quantities thus assume the role of primary elements of physical reality, with the result that this one is made up of events, and no longer objects.

The Hilbert space thus becomes the basic mathematical structure that allows the definition of events as projection operators on the one hand, and the specification of the conditional probability of causally related events on the other. This approach eliminates the fundamental role of measurement-based notions (such as that of 'observable') while retaining the potentiality of the conventional formalism. Detaching the meaning of quantum mechanical formalism from the narrow situation represented by the usual experimental setup with the stages of preparation, propagation and observation broadly extends the scope of application of formalism itself. The widespread use of formalism that has been made over the decades to describe the structure of matter (particles, nuclei, atoms, molecules, condensed states) is in this way justified.

The quantum jump is a non-unitary operation that converts quantum information (encoded in forward or backward amplitude) into classical information; an operation which is represented by an appropriate projector of a Hilbert space. The QJ consists in the temporal localization of the physical quantities summarized in this projector. "Matter" is understood here as the complex of these localizations and not as some kind of support for localized quantities. Each QJ is an elementary interaction in the course of which a quantum of action is exchanged and therefore materializes a single elementary cell (bit) in the phase space of a physical system. An analysis of the cosmological limits to positional information associated with these localizations was carried out in Section 5. It is important not to confuse this information with that processed by a quantum computer, as the Universe or a computer that simulates it. The cosmological limits induced on this second information are analyzed in Section 6 and the results are congruent with the well known calculation of Lloyd [64]. Differently from Lloyd's assumptions, the concept of "event", which is here distinct from that of "observation", does not imply in itself any irreversibility. As a result, most of the QJs are not registered.

Quantum information is processed between two successive QJs, causally connected through a transition amplitude. This processing does not "take place" in the usual sense of the term because the quantum superposition, considered in the time interval between the two QJs, is a pure mathematical construct (Section 2) that evolves only in the sense of its parametric dependence on the instant of the second QJ (forward amplitude) or first QJ (backward amplitude). Not surprisingly, the attempt to analyze this evolution in spatio-temporal terms leads to virtual trajectories joining virtual sub-events, for example the two paths in the double slit experience. These paths are naturally interfering and it has been argued that this is the origin of the hyper-Turing nature of quantum computing [65]. Taking this suggestion, an important field that opens to further investigations is that of the clarification of the relationships between the hyper-computational aspects of quantum computing and the non-local structure of elementary interactions modeled by quantum theories. The structure seems to indicate an emergent aspect of the spatio-temporal ordering of events.

Author Contributions: I.L. and L.C. equally contributed to the original idea and the writing of this manuscript.

Funding: This research was funded by the Research Institute for Astronomy and Astrophysics of Maragha (RIAAM) under research project No. 1/5750-65.

Acknowledgments: The authors dedicate this modest work to Francis David Peat, who has recently passed away. It was his words and overwhelming creativity in teaching us how space-time was too small to contain the world that inspired this work.

Conflicts of Interest: The authors declare no conflict of interest.

\section{References}

1. Plotnitsky, A. The Principles of Quantum Theory, from Planck's Quanta to the Higgs Boson: The Nature of Quantum Reality and the Spirit of Copenhagen; Springer: Berlin, Germany, 2016.

2. Bohr, N. On the constitution of atoms and molecules I. Philos. Mag. Ser. 1913, 6, 1-25. [CrossRef]

3. Bohr, N. On the constitution of atoms and molecules II. Philos. Mag. Ser. 1913, 6, 476-502. [CrossRef] 
4. Bohr, N. On the constitution of atoms and molecules III. Philos. Mag. Ser. 1913, 6, 857-875. [CrossRef]

5. Bergquist, J.C.; Hulet, R.G.; Itano, W.M.; Wineland, D.J. Observation of quantum jumps in a single atom. Phys. Rev. Lett. 1986, 57, 1699-1702. [CrossRef] [PubMed]

6. Nagourney, W.; Sandberg, J.; Demhelt, H. Shelved optical electron amplifier: Observation of quantum jumps. Phys. Rev. Lett. 1986, 56, 2797-2799. [CrossRef] [PubMed]

7. Sauter, T.; Neuhauser, W.; Blatt, R.; Toschek, P.E. Observation of quantum jumps. Phys. Rev. Lett. 1986, 57, 1696-1698. [CrossRef] [PubMed]

8. Blockley, C.A.; Walls, D.F.; Risken, H. Quantum collapses and revivals in a quantized trap. Europhys. Lett. 1992, 17, 509. [CrossRef]

9. Basche, T.; Kummer, S.; Brauchle, C. Direct spectroscopic observation of quantum jumps of a single molecule. Nature 1995, 373, 132-134. [CrossRef]

10. Peil, S.; Gabrielse, G. Observing the quantum limit of an electron cyclotron: QND measurements of quantum jumps between Fock states. Phys. Rev. Lett. 1999, 83, 1287-1290. [CrossRef]

11. Gleyzes, S.; Kuhr, S.; Guerlin, C.; Bernu, J.; Deléglise, S.; Hoff, U.B.; Brune, M.; Raimond, J-M.; Haroche, S. Quantum jumps of light recording the birth and death of a photon in a cavity. Nature 2007, 446, 297-300. [CrossRef]

12. Yu, Y.; Zhu, S-L.; Sun, G.; Wen, X.; Dong, N.; Chen, J.; Wu, P.; Han, S. Quantum jumps between macroscopic quantum states of a superconducting qubit coupled to a microscopic two-level system. Phys. Rev. Lett. 2008, 101, 157001. [CrossRef] [PubMed]

13. Reick, S.; Mølmer, K.; Alt, W.; Eckstein, M.; Kampschulte, T.; Kong, L.; Reimann, R.; Thobe, A.; Widera, A.; Meschede, D. Analyzing quantum jumps of one and two atoms strongly coupled to an optical cavity. J. Opt. Soc. Am. B 2010, 27, A152-A163. [CrossRef]

14. Neumann, P.; Beck, J.; Steiner, M.; Rempp, F.; Fedder, H.; Hemmer, P.R.; Wrachtrup, J.; Jelezko, F. Single-shot readout of a single nuclear spin. Science 2010, 329, 542-544. [CrossRef] [PubMed]

15. Vamivakas, A.N.; Lu, C-Y.; Matthiesen, C.; Zhao, Y.; Falt, S.; Badolato, A.; Atatüre, M. Observation of spin-dependent quantum jumps via quantum dot resonance fluorescence. Nature 2010, 467, 297-300. [CrossRef] [PubMed]

16. Vijai, R.; Slichter, D.H.; Siddiqi, I. Observation of quantum jumps in a superconducting artificial atom. Phys. Rev. Lett. 2011, 106, 110502. [CrossRef] [PubMed]

17. Ulmer, S.; Rodegheri, C.C.; Blaum, K.; Kracke, H.; Mooser, A.; Quint, W.; Walz, J. Observation of spin flips with a single trapped proton. Phys. Rev. Lett. 2011, 106, 253001. [CrossRef]

18. Murch, K.W.; Weber, S.J.; Macklin, C.; Siddiqi, I. Observing single quantum trajectories of a superconducting quantum bit. Nature 2013, 502, 211-214. [CrossRef]

19. Wolf, F.; Wan, Y.; Heip, J.C.; Gebert, F.; Shi, C.; Schmidt, P.O. Non-destructive state detection for quantum logic spectroscopy of molecular ions. Nature 2016, 530, 457. [CrossRef]

20. Davies, P.C.W. The implications of a cosmological information bound for complexity, quantum information and the nature of physical law. In Randomness and Complexity, from Leibniz to Chaitin; Calude, C.S., Ed.; World Scientific: Singapore, 2007; pp. 69-88.

21. Aaronson, S. Quantum computing since Democritus; Cambridge University Press: Cambridge, UK, 2013.

22. Licata, I.; Fiscaletti, D. Bohm trajectories and Feynman paths in light of quantum entropy. Acta Phys. Pol. B 2014, 45, 885-904. [CrossRef]

23. Aerts, D.; Aerts, S.; Durt, T.; Leveque, O. Classical and quantum probability in the epsilon model. Int. J. Theor. Phys. 1999, 38, 407-429. [CrossRef]

24. Aerts, S. The Born rule from a consistency requirement on hidden measurements in complex Hilbert space. Int. J. Theor. Phys. 2005, 44, 999-1009. [CrossRef]

25. Chiatti, L.; Licata, I. Relativity with respect to measurement: Collapse and quantum events from Fock to Cramer. Systems 2014, 2, 576-589. [CrossRef]

26. Chiatti, L. The transaction as a quantum concept. Int. J. Res. Rev. Appl. Sci. 2013, 16, $28-47$.

27. Schrödinger, E. Die gegenwärtige situation in der quantenmechanik. Naturwissenschaften 1935, 23, 807-812, 823-828, 844-849. [CrossRef]

28. Wigner, E.P. Remarks on the mind-body question. In Symmetries and Reflections; Indiana University Press: Bloomington, IN, USA, 1967; pp. 171-184. 
29. Frauchiger, D.; Renner, R. Quantum theory cannot consistently describe the use of itself. Nat. Commun. 2018, 9, 3711. [CrossRef]

30. Bekenstein, J.D. How does the entropy/information bound work? Found. Phys. 2005, 35, 1805-1823. [CrossRef]

31. Chiatti, L.; Licata, I. Fluidodynamical representation and quantum jumps. In Quantum Structural Studies. Classical Emergence from the Quantum Level; Kastner, R.E., Jeknic-Dugic, J., Eds.; World Scientific: Singapore, 2017; pp. 201-223.

32. Rozema, L.A.; Darabi, A.; Mahler, D.H.; Hayat, A.; Soudagar, Y.; Steinberg, A.M. Violation of Heisenberg's measurement-disturbance relationship by weak measurements. Phys. Rev. Lett. 2012, 109, 189902. [CrossRef]

33. Bekenstein, J.D. Black holes and entropy. Phys. Rev. D 1973, 7, 2333-2346. [CrossRef]

34. Bohm, D.; Hiley, B.J. Measurement understood through the quantum potential approach. Found. Phys. 1984, 14, 255-274. [CrossRef]

35. Licata, I. Emergence to the edge between classical and quantum information. In Physics of Emergence and Organization; Licata, I., Sakaji, A., Eds.; World Scientific: Singapore, 2008; pp. 1-25.

36. Hiley, B. Bohmian non-commutative dynamics: History and new developments. arXiv 2013, arXiv:1303.6057v1.

37. Hiley, B.; Monk, N. Quantum phase space and the discrete Weyl algebra. Mod. Phys. Lett. A 1993, $08,3625$. [CrossRef]

38. Rovelli, C. Quantum mechanics without time: A model. Phys. Rev. D 1990, 42, 2638-2646. [CrossRef]

39. Licata, I. In and out of screen. On some new considerations about localization and delocalization in archaic theory. In Beyond Peaceful Coexistence. The Emergence of Space, Time and Quantum; Licata, I., Ed.; Imperial College Press: Cambridge, UK, 2016; pp. 559-578.

40. Caldirola, P. On a relativistic model of the electron. Nuovo Cimento A 1979, 49, 497-611. [CrossRef]

41. Ghirardi, G.C.; Rimini, A.; Weber, T. Unified dynamics for microscopic and macroscopic systems. Phys. Rev. D 1986, 34, 470. [CrossRef]

42. Ghirardi, G.C.; Pearle, P.; Rimini, A. Markov processes in Hilbert space and continuous spontaneous localizations of systems of identical particles. Phys. Rev. A 1980, 42, 78. [CrossRef]

43. Ghirardi, G.C.; Grassi, R.; Benatti, F. Describing the macroscopic world: Closing the circle within the dynamical reduction program. Found. Phys. 1995, 25, 5-38. [CrossRef]

44. Tumulka, R. A relativistic version of the Ghirardi-Rimini-Weber model. J. Stat. Phys. 2006, 125, 821-840. [CrossRef]

45. Bell, J.S. Are there quantum jumps? In Schrödinger. Centenary Celebration of a Polymath; Kilmister, C.W., Ed.; Cambridge University Press: Cambridge, UK, 1987; pp. 41-52.

46. Esfeld, M.; Deckert, D. A. A Minimalist Ontology of the Natural World; Routledge Studies in the Philosophy of Mathematics and Physics; Routledge: London, UK, 2017.

47. Esfeld, M.; Gisin, N. The GRW flash theory: A relativistic quantum ontology of matter in space-time? Philos. Sci. 2014, 81, 248-264. [CrossRef]

48. Vigier, J.P. Structure of Micro-Objects in the Causal Interpretation of Quantum Theory; Gauthier-Villars: Paris, France, 1956.

49. Castro, P.; Gatta, M.; Croca, J.R.; Moreira, R. Spacetime as an emergent phenomenon: A possible way to explain entanglement and the tunnel effect. J. Appl. Math. Phys. 2018, 6, 2107-2118. [CrossRef]

50. Cramer, J.G. Generalized absorber theory and the Einstein-Podolsky-Rosen paradox. Phys. Rev. D 1980, 22, 362-376. [CrossRef]

51. Cramer, J.G. The arrow of electromagnetic time and generalized absorber theory. Found. Phys. 1983, 13, 887-902. [CrossRef]

52. Cramer, J.G. The transactional interpretation of quantum mechanics. Rev. Mod. Phys. 1986, 58, 647-687. [CrossRef]

53. Kastner, R.E. The Transactional Interpretation of Quantum Mechanics: The Reality of Possibility; Cambridge University Press: Cambridge, UK, 2012.

54. Chiatti, L. The Archetypal Structures of the Physical World; Di Renzo: Rome, Italy, 2005. (In Italian)

55. Kastner, R.E.; Cramer, J.G. Quantifying absorption in the transactional interpretation. Int. J. Q. Found. 2018, 4, 210-222.

56. Bohm, D.; Hiley, B.J. The Undivided Universe; Routledge: London, UK, 1993.

57. Zeh, H.D. On the interpretation of measurement in quantum theory. Found. Phys. 1970, 1, 69-76. [CrossRef] 
58. Zurek, W.H. Pointer basis of quantum apparatus: Into what mixture does the wave packet collapse? Phys. Rev. D 1981, 24, 1516. [CrossRef]

59. Zurek, W.H. Environment-induced superselection rules. Phys. Rev. D 1982, 26, 1862. [CrossRef]

60. Zurek, W.H. Decoherence, einselection, and the quantum origins of the classical. Rev. Mod. Phys. 2003, 75, 715. [CrossRef]

61. Schlosshauer, M. Decoherence, the measurement problem, and interpretations of quantum mechanics. Rev. Mod. Phys. 2005, 76, 1267. [CrossRef]

62. Schlosshauer, M. Decoherence and the Quantum-to-Classical Transition; Springer: Berlin, Germany, 2007.

63. Simonius, M. Spontaneous symmetry breaking and blocking of metastable states. Phys. Rev. Lett. 1978, 40, 980-983. [CrossRef]

64. Loyd, S. Computational capacity of the Universe. Phys. Rev. Lett. 2002, 88, 237901. [CrossRef]

65. Licata, I. Effective physical processes and active information in quantum computing. In New Trends in Quantum Information; Sakaji, A., Licata, I., Singh, J., Felloni, S., Eds.; Aracne: Rome, Italy, 2010; pp. 15-32.

(C) 2019 by the authors. Licensee MDPI, Basel, Switzerland. This article is an open access article distributed under the terms and conditions of the Creative Commons Attribution (CC BY) license (http:// creativecommons.org/licenses/by/4.0/). 